\title{
Special issue on fog/edge computing in Enterprise Multimedia Security [SI 1138T]
}

\author{
Victor Chang ${ }^{1} \cdot$ Gang Sun $^{2} \cdot$ Gary Wills $^{3}$ \\ Published online: 3 February 2020 \\ (C) Springer Science+Business Media, LLC, part of Springer Nature 2020
}

Enterprise Multimedia Security (EMS) is the key to achieving global information security in business and organizations. Fog/Edge computing is a new paradigm for enterprise where businesses need to be secured. However, this new trend needs to be more systematic concerning fog/edge multimedia security, which is a factor in sustaining cloud technology by the building-in trust. For example, current challenges with cybersecurity and application security flaws are highlighting important lessons to be learned and also lead to the adoption of best practices. Similarly, as the demand for multimedia services increases, the importance of security and privacy is always on the way up. We oversee the importance of enterprise security as a unique and rising field to ensure all aspects of security and risks can be identified, surveyed, tested, prototyped and minimized with recommendations and lessons learned disseminated. The scope of enterprise multimedia security has expanded into risk management/analysis, management of future technologies such as internet of things and big data and modern ethical hacking methods with multimedia. We need to provide a robust and enhanced level of security. As a result, we seek high-quality papers demonstrating proofs-of-concept, prototype and successful implementation for fog and edge computing in EMS.

We are honored and glad that we received 32 submissions since the opening of the call for papers in November 2018. We went through vigorous review processes. Experts in security and privacy domains were active and keen to provide us recommendations. The guest editor team discussed internally to maintain high-quality assurance for our scholarly selections. We ensured that only papers demonstrating real research contributions were considered. In the end,

Victor Chang

V.Chang@tees.ac.uk

Gang Sun

gangsun@uestc.edu.cn

Gary Wills

gbw@ecs.soton.ac.uk

1 School of Computing, Engineering and Digital Technologies, Teesside University, Middlesbrough, UK

2 University of Electronic Science and Technology of China (UESTC), Chengdu, Sichuan, China

3 Electronics and Computer Science, University of Southampton, Southampton, UK 
only eight papers were selected for our special issue (SI) before February 2020. We completed vigorous review processes even before our scheduled deadline, which was considered a remarkable achievement for guest editors. We have also maintained a precisely $25 \%$ acceptance rate.

This SI has brought scientists, professors, and practitioners together. Each selected paper has very solid theoretical and empirical research contributions validated by theoretical proof, simulations, experiments and quantitative analysis. This was one of the first few completed SIs under Prof. Victor Chang's management when he became a Full Professor in September 2019. This SI could also bring us great luck.

It is the tradition for the guest editors not to mention much in this editorial note. But we would sincerely ask you to enjoy every selected paper in this SI.

We are grateful to the MTAP editorial team and the Editor-in-Chief to provide us the opportunity to serve this SI for several research communities, since this topic is within their common interests. We hope that we have the opportunity again in the near future!

Publisher's note Springer Nature remains neutral with regard to jurisdictional claims in published maps and institutional affiliations. 